\title{
Hybrid Algorithm for Dose Calculation in Cms Xio Treatment Planning System
}

\author{
Akpochafor M. O.,Aweda M. A., Durosinmi-Etti F.A., Adeneye S.O. \\ Department of Radiation Biology, Radiotherapy, Radiodiagnosis and Radiography, \\ College of Medicine/Lagos University Teaching Hospital, PMB 12003, Lagos, Nigeria.
}

\begin{abstract}
This study aimed at designing an improved hybrid algorithm by explicitly solving the linearized Boltzmann transport equation (LBTE) which is the governing equation that describes the macroscopic behaviour of radiation particles (neutrons, photons, electrons, etc). The algorithm accuracy will be evaluated using a newly designed in-house verification phantom and its results will be compared to those of the other XiO photon algorithms. The LBTE was solved numerically to compute photon transport in a medium. A programming code (algorithm) for the LBTE solution was developed and applied in the treatment planning system (TPS). The accuracy of the algorithm was evaluated by creating several plans for both the designed phantom and solid water phantom using the designed algorithm and other Xio photon algorithms. The plans were sent to a pre-calibrated Eleckta linear accelerator for measurement of absorbed dose. The results for all treatment plans using the hybrid algorithm compared to the 3 Xio photon algorithms were within $4 \%$ limit. Calculation time for the hybrid algorithm was less in plans with larger number of beams compared to the other algorithms; however, it is higher for single beam plans. The hybrid algorithm provides comparable accuracy in treatment planning conditions to the other algorithms. This algorithm can therefore be employed in the calculation of dose in advance techniques such as IMRT and Rapid Arc by a radiotherapy centres with cmsxio treatment planning system as it is easy to implement.
\end{abstract}

\section{Introduction}

The knowledge of radiation dose distribution in the patient is required before they undergo radiotherapy treatment. This can be ascertained by planning the treatment procedure on a dedicated computer system (treatment planning). Treatment planning can be described as the iterative process whereby the treatment strategy of the oncologist is quantified as a set of instructions including a description of the expected dose distribution in the patient. Planning is based on predictions of dosage delivered to the patient by the proposed arrangement of radiation beams. When a treatment planning system (TPS) is commissioned, beam data from local linear accelerator must be entered into it. The data requirements for computerised treatment planning systems may be categorised in terms of how the data will be used. It is necessary for the TPS to represent each individual treatment machine. The details and format of the required data will depend on the requirements of each planning system. Treatment planning will almost certainly include measured data and user-determined data. It must also be verified that any averaging of data by the TPS algorithms does not produce unacceptable differences from the measured data. The efficiency of the calculation algorithm in both standard and nonstandard conditions must be tested to determine its applicability and limitations. The distinction must be made between the data collected for entry into the TPS and data collected in order to validate the TPS. Basic data are entered into and used by the TPS to calculate dose distributions. Reference data are acquired under standard conditions (usually in water-filled plotted tank) to validate basic data or check the TPS algorithm. After the full commissioning process of the TPS, verification of its accuracy is often done by using a verification phantom, an object that mimics human body that can be used for planning and treated like a normal patient.

There are several algorithms in the treatment planning system that plays different roles. However, dose calculation algorithms play the central role of calculating dose within the patient while considering the beam parameters (Van Dyk, 1993). Algorithms are asequence of instructions that operate on a set of input data, transforming the information into a set of output results that are of interest to the user (Animesh, 2005).For every algorithm, the precision of the dose distribution depends on the data or parameters used by the algorithm and its assumptions. Examples of commercially available algorithms includes, anisotropic analytic algorithm, fast furrier transform convolution algorithm, superposition algorithm, collapse cone convolution, Monte Carlo program, fast superposition, inhomogeneous correction algorithm, modified Clarkson sector integration, area integral algorithm, etc.

The accuracy with which algorithms are able to predict dose distribution is dependent upon the assumptions and approximations that the algorithms makes. Also, the speed of calculation of monitor units is highly dependent on the number of fields in a treatment plan for most algorithms. The verification of the accuracy and the speed of these algorithms using heterogeneous phantom for measurement is important. An ideal 
algorithm is one which has good compromise between precision and speed in an inhomogeneous medium. Majority of the commercially available algorithms lack this quality and hence, the need for this study.

The CMS XiO treatment planning system which contains Clarkson, fast furrier transform (FFT) convolution, Superposition, Faster superposition, and Electron 3D pencil beam algorithms are used for the calculation of photon and electron dose distribution. Each of the algorithms has its limitations regarding the balance between speed and precision of calculation and as such, a need for an improved algorithm that can reduce the time of calculation with improved precision in dose calculation where multiple fields and larger monitor units are involved is important. This is essential for advanced radiotherapy techniques such as intensity modulated radiotherapy that includes many fields and large monitor units.

\section{Methods and Material}

The Boltzmann transport equation (BTE) is the governing equation which describes the macroscopic behaviour of radiation particles (photons, electrons, neutrons, protons, etc.) as they travel through and interact with matter. The Linear Boltzmann transport equation (LBTE) is a form of the BTE which assumes that radiation particles only interact with the matter they are passing throughand not with each other: this is valid for conditions without external magnetic fields. There are different ways of solving the LBTE; however, the numerical method proposed by Lewis et al., (Lewis et al., 1984) is the method that can be used to solve the equation explicitly.The LTBE was solved using a similar method applied byVassilievet al., (Vassiliev et al., 2010):

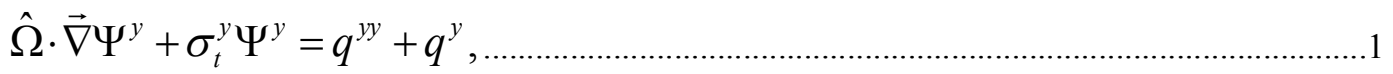

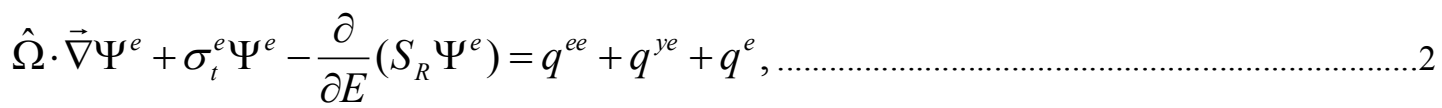

The first term on the left hand side of equations 1 and 2 is the streaming operator. The second term on the left hand side of equations 1 and 2 is the collision or removal operator. Equation 2 is the Boltzmann FokkerPlanck transport equation (Wareing et al., 2000, Wareing et al., 2001), which is solved for the electron transport. In Equation 2, the third term on the left represents the continuous slowing down (CSD) operator, which accounts for Coulomb 'soft' electron collisions. The right hand side of Equations 1 and 2 include the scattering, production, and the external source terms $\left(q^{y}\right.$ and $\left.q^{e}\right)$. The scattering and production sources are defined by:

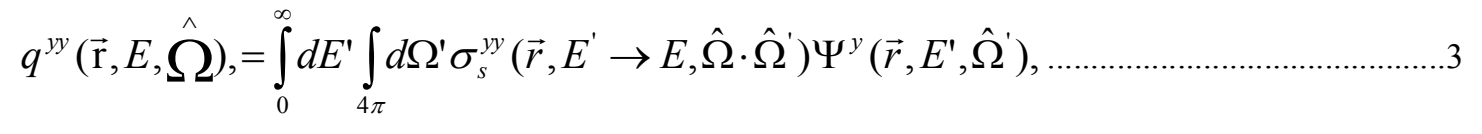

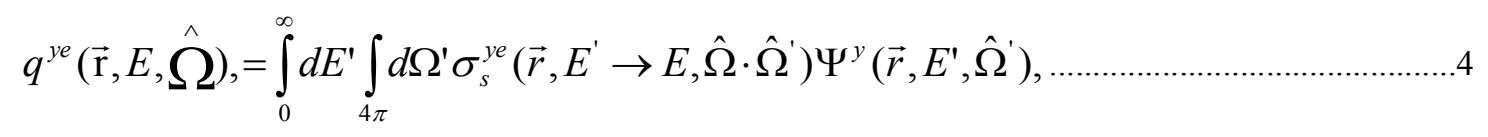

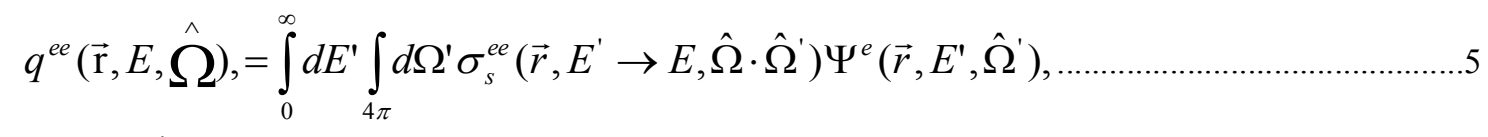

$$
\begin{aligned}
& \text { Where } \\
& \sigma_{s}^{y y}=\text { Macroscopic photon-to-photon differencial scattering cross section } \\
& \sigma_{s}^{y e}=\text { Macroscopic photon-to-electron differencial production cross section } \\
& \sigma_{s}^{e e}=\text { Macroscopic electron-to-electron differencial scattering cross section }
\end{aligned}
$$

The following equation represents the un-collided photon fluence:

$$
\hat{\Omega} \cdot \vec{\nabla} \Psi_{u n c}^{y}+\sigma_{t}^{y} \Psi_{u n c}^{y}=q^{y}(E, \hat{\Omega}) \delta\left(\vec{r}-\vec{r}_{p}\right),
$$

A property of Equation 6 was that $\vec{\nabla} \Psi_{u n c}^{y}$ can be solved for analytically. Doing so provides the following expression for the un-collided photon angular fluence from a point source: 


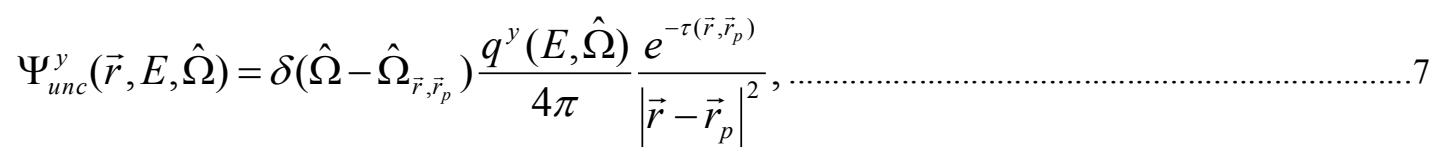

where,

$\hat{\Omega}_{\vec{r}, \vec{r}_{p}}=\frac{\left|\vec{r}_{p}-\vec{r}\right|}{\left|\vec{r}-\vec{r}_{p}\right|}$, and $\vec{r}_{p}$ and $\vec{r}$ are the source and destination points of the ray trace, respectively.

$\tau\left(\vec{r}-\vec{r}_{p}\right)=$ The optical distance (measured in mean-free-paths) between $\vec{r}$ and $\vec{r}_{p}$.

Once the electron angular fluencewas solved for all energy groups, the dose in any output grid voxel was obtained through the following equation proposed bySiebers et al. (Siebers et al., 2000):

$$
D_{i}=\int_{0}^{\infty} d E \int_{4 \pi} d \hat{\Omega} \frac{\sigma_{E D}^{e}(\vec{r}, E)}{\rho(\vec{r})} \Psi^{e}(\vec{r}, E, \vec{\Omega}),
$$

Where,

$\sigma_{E D}^{e}=$ macroscopic electron energy deposition cross sections (in $\mathrm{MeV} / \mathrm{cm}$ )

$\rho=$ Material density (in $\mathrm{g} / \mathrm{cm}^{3}$ )

The iteration scheme used to solve the equations is shown in the algorithm below:

\section{The algorithm}

$\%$ File: Linear Boltzmann Equations

$\%$ Date: 12 th of March 2012

$\%$ Author: Michael Akpochafor

$\%$ the equation here perform time independent single calculation at high resolutions

$\% \mathrm{D}(\operatorname{vector}(\mathrm{r}))=\operatorname{lint}(\mathrm{mu} / \mathrm{P})^{*}(\mathrm{psi}) \_\mathrm{p}\left\{\right.$ vector $\left.(\mathrm{r})^{\prime *} \mathrm{~A}^{*}\left[\operatorname{vector}(\mathrm{r})-\operatorname{vector}(\mathrm{r})^{\prime}\right]^{*} \mathrm{~d}^{\wedge} 3 *\left(\operatorname{vector}(\mathrm{r})^{\prime}\right)\right\}$

$\% \mathrm{D}($ vector $(\mathrm{r}))=$ dose at a point

$\%(\mathrm{mu} / \mathrm{P})=$ mass attenuation coefficient

$\%(\mathrm{psi}) \_$p $\left\{\right.$vector $(\mathrm{r})^{\prime}=$ primary photon energy fluence

$\% \mathrm{~A}^{*}\left[\operatorname{vector}(\mathrm{r})\right.$-vector $\left.(\mathrm{r})^{\prime}\right]=$ convolution kernel, the distribution of fraction energy Imparted per unit volume.

$\left.\%(\text { vector }(\mathrm{r}))^{\prime}\right)=$ TERMA at depth includes the energy retained by the photon.

$\%$ Plots a Linearized Boltzmann distribution Equations

$\%$ for dose calculation.

$\%$ THIS PROGRAMME SOLVE THE EQUATION (8)

$\% \mathrm{D}(\mathrm{i})=$ int_0 ${ }^{\wedge}$ inffy $* \mathrm{dE} *$ int_4*pi ${ }^{\wedge}$ inffy $* \mathrm{~d}\left({ }^{\prime}\right.$ omega $)$ vector $* \backslash$ frac $\backslash$ sigma_ED

$\%{ }^{\wedge} \mathrm{e}(\mathrm{r}($ vector $), \mathrm{E}) /$ rho(vector)* ${ }^{*}(\text { vector })^{*} \backslash \mathrm{psi} \mathrm{p}^{\wedge} \mathrm{e}(\mathrm{r}, \mathrm{E}$, lomega(all vector $\left.)\right)$

endTime $=5000$

tlist $=0: 50$ :endTime;

numNodes $=\operatorname{size}(\mathrm{p}, 2)$;

$\%$ Set the initial temperature of all nodes to ambient, $300 \mathrm{~K}$

u0 (1:numNodes $)=300$;

$\%$ Find all nodes along the bottom edge and set their initial temperature

$\%$ to the value of the constant $\mathrm{BC}, 1000 \mathrm{~K}$

$\operatorname{nodes} \mathrm{Y0}=\operatorname{abs}(\mathrm{p}(2,:))<1.0 \mathrm{e}-5$;

$\mathrm{u} 0($ nodesY0) $=1000$;

rtol $=1.0 \mathrm{e}-3 ;$ atol $=1.0 \mathrm{e}-4$

$\%$ The transient solver parabolic automatically handles both linear

$\%$ and nonlinear problems, such as this one.

$\mathrm{u}=$ parabolic(u0, tlist, b,p,e,t,c,a,f,d,rtol,atol);

figure;

plot (tlist, $\mathrm{u}(3, \mathrm{~s}))$; grid;

title 'Temperature Along the Top Edge of the Plate as a Function of Time'

xlabel 'Time, seconds'

ylabel 'Temperature, degrees-Kelvin'

\%figure; 
pdeplot(p, e, t, 'xydata', u(:,end), 'contour', 'on', 'colormap', 'jet');

title(sprintf('Temperature In The Plate, Transient Solution( \%d seconds) $\backslash \mathrm{n}$ ', ...

tlist $(1$, end $))$ );

xlabel 'X-coordinate, meters'

ylabel 'Y-coordinate, meters'

$\%$ fprintf('InTemperature at the top edge of the plate $(\mathrm{t}=\% 5.1 \mathrm{f}$ secs $)=\% 5.1 \mathrm{f}$ degrees-K $\ln$ ', ...

tlist (1,end), $u(4$, end $)$ );

\section{Measurement of absorbed dose using the hybrid algorithm and other cmsxio algorithms}

Treatment plans weredesigned toprescribe $1.0 \mathrm{~Gy}$ at the Iso-centre using the hybrid algorithm and other cmsxio algorithms. The time of calculation of absorbed dose for the different algorithms was recorded for several treatment plans. The plans were transferred to the pre-calibrated ELEKTA-Precise clinical linear accelerator (Eleckta Oncology System, 2000) for measurements.

Measurements were carried out with $6 \mathrm{MeV}$ photon beams from the ELEKTA-Precise clinical linear accelerator using an iso-centric set up as shown in figures $1 \mathrm{a}$ and $\mathrm{b}$ respectively. A pre-calibrated farmer-type ionization chamber along with its electrometer (figure 2) was used to measure the absorbed dose delivered. The ionization chamber was calibrated by cross calibrating it against a reference ionization chamber to obtain the calibration factor. Measurements were taken at the depth of $12 \mathrm{~cm}$ on the solid water; a depth corresponding to the point where the ionisation chamber is placed on the phantom. Six measurements were made for each treatment plan using the different algorithms for comparison. The absorbed dose at the reference depth was determined using the IAEA TRS 398 protocol (IAEA, 2000) described in equation 9 below:

The absorbed dose at reference depthwas calculated as follows:

$$
\mathbf{D}_{\mathbf{w}, \mathbf{Q}}=\mathbf{M}_{\mathbf{Q}} \times \mathbf{N}_{\mathbf{D}, \mathbf{w}, \mathbf{x}} \mathbf{k}_{\mathbf{Q}, \mathbf{Q}}
$$

where $\mathrm{M}_{\mathrm{Q}}$ is the electrometer response corrected for temperature and pressure.

$\mathrm{N}_{\mathrm{D}, \mathrm{w}}$ is the chamber calibration factor andk $\mathrm{k}_{\mathrm{Q}, \mathrm{Q}}$ is the factor which corrects for difference in the response of the dosimeter at the calibration quality $\mathrm{Q}_{0}$ and at quality $\mathrm{Q}$ of the clinical x-ray beam according to the TRS 398 protocol of the IAEA (IAEA, 2000). Calculated dose at Dmax was compared to the expected dose of $1 \mathrm{~Gy}$.

Deviation between expected and measured dose was obtained using the relation:

$\%$ Deviation $=D_{\text {meas }}-D_{\text {ref }} \times \mathbf{1 0 0}$.

$\mathbf{D}_{\text {ref }}$

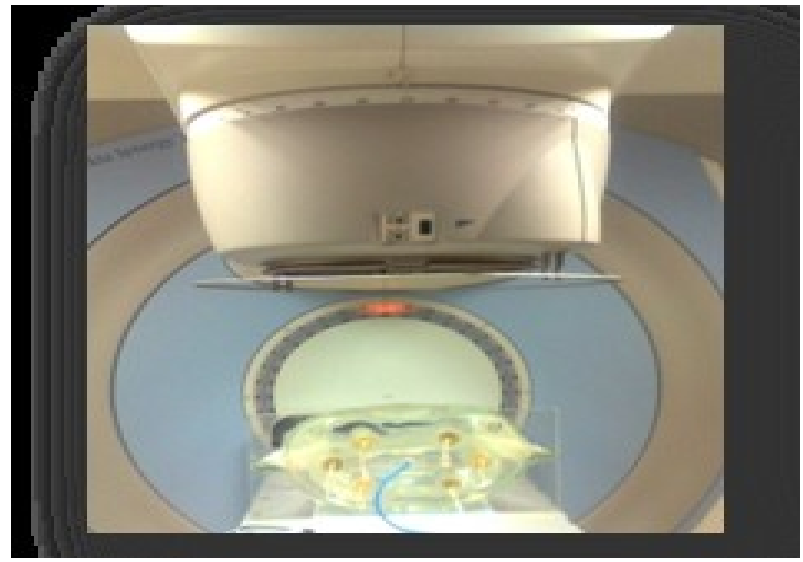

Fig 1a: Isocentric set up with the designed phantom

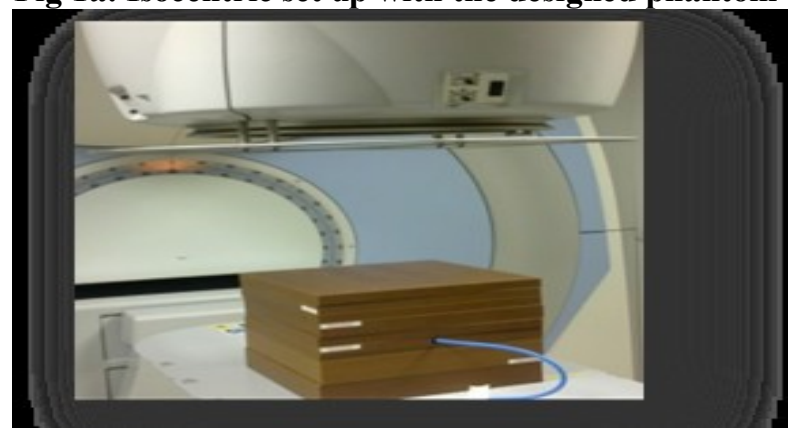

Fig. 1 b: The solid water phantom set-up under linear accelerator. 


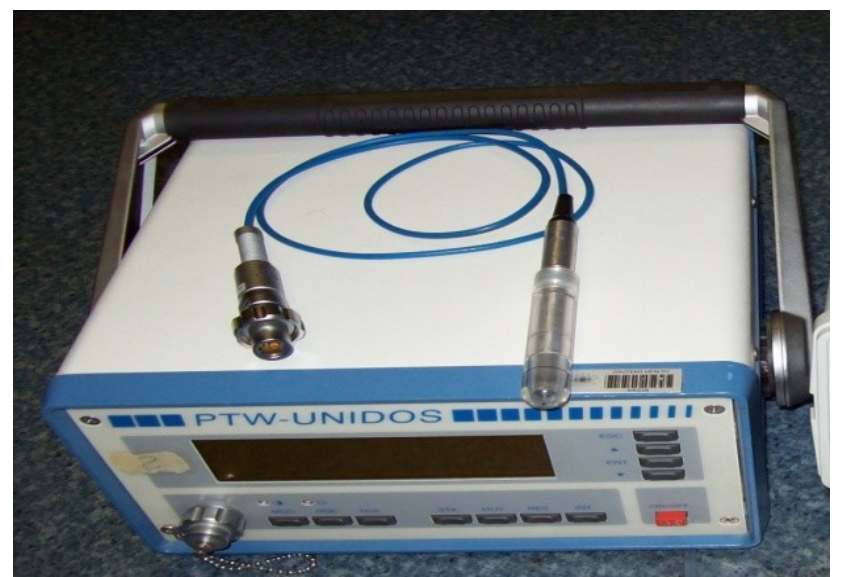

Fig. 2: Farmer type ionization chamber along with its electrometer.

\section{Results}

Absorbed dose measured at the LINAC for $6 \mathrm{MeV}$ photon beam using the different algorithms

Table 1: Comparison of absorbed doses (Gy) in solid water phantom for (a) Single field (b) Wedged field (c) Oblique fields (d) Oppose fields (e) Three fields (f) four fields (g) 6 fields (h) 9 fields and (i) 12 fields for different algorithms
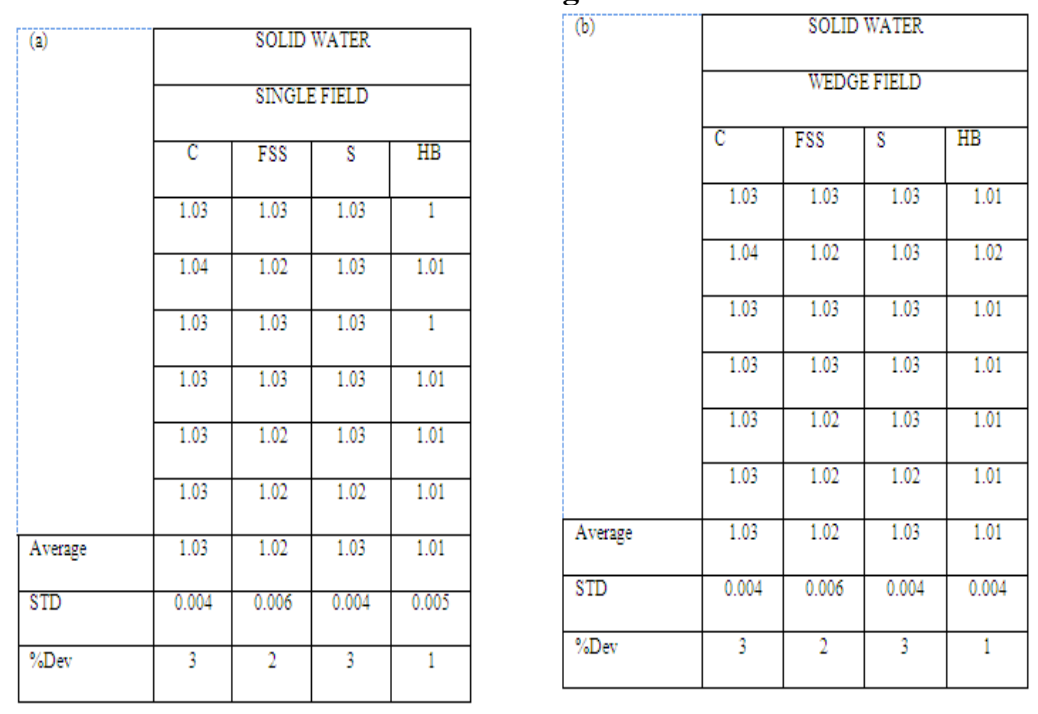

\begin{tabular}{|c|c|c|c|c|}
\cline { 2 - 5 }$(\mathrm{c})$ & \multicolumn{4}{c|}{ SOLID WATER } \\
\cline { 2 - 5 } & \multicolumn{4}{|c|}{ OBLIQUE FIELDS } \\
\cline { 2 - 5 } & $\mathrm{C}$ & FSS & $\mathrm{S}$ & HB \\
\cline { 2 - 5 } & 1.01 & 1.02 & 1.01 & 1.01 \\
\cline { 2 - 5 } & 1.02 & 1.01 & 1.02 & 1 \\
\cline { 2 - 5 } & 1.01 & 1.02 & 1.01 & 1 \\
\cline { 2 - 5 } & 1.01 & 1.02 & 1.01 & 1 \\
\cline { 2 - 5 } & 1.01 & 1.02 & 1.01 & 1 \\
\cline { 2 - 5 } & 1.01 & 1.01 & 1.02 & 1 \\
\hline Average & 1.01 & 1.02 & 1.01 & 1 \\
\hline STD & 0.004 & 0.005 & 0.005 & 0.004 \\
\hline \%Dev & 1 & 2 & 1 & 0 \\
\hline
\end{tabular}

\begin{tabular}{|c|c|c|c|c|}
\cline { 2 - 5 } (d) & \multicolumn{4}{c|}{ SOLID WATER } \\
\cline { 2 - 5 } \multicolumn{1}{c|}{} & \multicolumn{4}{c|}{ OPOSSING FIELDS } \\
\cline { 2 - 5 } & $\mathrm{C}$ & FSS & $\mathrm{S}$ & HB \\
\cline { 2 - 5 } & 1 & 0.98 & 1 & 1 \\
\cline { 2 - 5 } & 0.99 & 0.99 & 0.99 & 1.01 \\
\cline { 2 - 5 } & 0.98 & 0.98 & 1 & 1.01 \\
\cline { 2 - 5 } & 1 & 0.98 & 1 & 1.01 \\
\cline { 2 - 5 } & 0.99 & 0.98 & 0.99 & 1 \\
\cline { 2 - 5 } & 1 & 0.98 & 1 & 1.01 \\
\hline Average & 0.99 & 0.98 & 1 & 1.01 \\
\hline STD & 0.006 & 0.004 & 0.005 & 0.005 \\
\hline \%Dev & -0.1 & -2 & 0 & 1 \\
\hline
\end{tabular}


Hybrid Algorithm For Dose Calculation In Cms Xio Treatment Planning System

\begin{tabular}{|l|c|c|c|c|}
\cline { 2 - 5 }$(\mathrm{e})$ & \multicolumn{4}{|c|}{ SOLID WATER } \\
\cline { 2 - 5 } \multicolumn{1}{c|}{} & \multicolumn{4}{|c|}{ THREE FIELDS } \\
\cline { 2 - 5 } & $\mathrm{C}$ & FSS & $\mathrm{S}$ & HB \\
\cline { 2 - 5 } & 1.03 & 1.02 & 1.02 & 1 \\
\cline { 2 - 5 } & 1.02 & 1.01 & 1 & 1 \\
\cline { 2 - 5 } & 1.02 & 1 & 1.01 & 1 \\
\cline { 2 - 5 } & 1.03 & 1.02 & 1.01 & 1.01 \\
\cline { 2 - 5 } \multicolumn{1}{c|}{} & 1.02 & 1 & 1.01 & 1 \\
\cline { 2 - 5 } & 1.02 & 1.01 & 1 & 1 \\
\hline Average & 1.02 & 1.01 & 1.01 & 1 \\
\hline STD & 0.005 & 0.009 & 0.008 & 0.004 \\
\hline \%Dev & 2 & 1 & 1 & 0 \\
\hline
\end{tabular}

\begin{tabular}{|l|c|c|c|c|}
\cline { 2 - 5 } (f) & \multicolumn{4}{c|}{ SOLID WATER } \\
\cline { 2 - 5 } \multicolumn{1}{c|}{} & \multicolumn{4}{c|}{ 4 FIELDS PLAN } \\
\cline { 2 - 5 } & $\mathrm{C}$ & FSS & $\mathrm{S}$ & HB \\
\cline { 2 - 5 } & 1.02 & 1.01 & 1.02 & 1.01 \\
\cline { 2 - 5 } & 1.02 & 1.02 & 1.02 & 1.01 \\
\cline { 2 - 5 } & 1.02 & 1.02 & 1.02 & 1.01 \\
\cline { 2 - 5 } & 1.02 & 1.01 & 1.02 & 1.01 \\
\cline { 2 - 5 } & 1.02 & 1.01 & 1.02 & 1 \\
\cline { 2 - 5 } & 1.02 & 1.02 & 1.02 & 1.01 \\
\hline Average & 1.02 & 1.01 & 1.02 & 1 \\
\hline STD & 0 & 0.005 & 0 & 0.005 \\
\hline \%Dev & 2 & 1 & 2 & 1 \\
\hline
\end{tabular}

\begin{tabular}{|l|c|c|c|c|}
\cline { 2 - 5 }$(\mathrm{g})$ & \multicolumn{4}{c|}{ SOLID WATER } \\
\cline { 2 - 5 } \multicolumn{1}{c|}{} & \multicolumn{4}{c|}{6 FIELD PLAN } \\
\cline { 2 - 5 } & $\mathrm{C}$ & FSS & $\mathrm{S}$ & HB \\
\cline { 2 - 5 } & 1.01 & 1.02 & 1.01 & 1.01 \\
\cline { 2 - 5 } & 1 & 1.01 & 1 & 1.01 \\
\cline { 2 - 5 } \multicolumn{1}{c|}{} & 1 & 1.01 & 1 & 1.01 \\
\cline { 2 - 5 } & 1 & 1.01 & 1.01 & 1 \\
\cline { 2 - 5 } & 1 & 1.01 & 1.01 & 1.01 \\
\cline { 2 - 5 } & 1 & 1.01 & 1.01 & 1.01 \\
\hline Average & 1 & 1.01 & 1.01 & 1.01 \\
\hline STD & 0.004 & 0.004 & 0.005 & 0.004 \\
\hline \%Dev & 0 & 1 & 1 & 1 \\
\hline
\end{tabular}

\begin{tabular}{|l|c|c|c|c|}
\cline { 2 - 5 } \multicolumn{1}{c|}{} & \multicolumn{4}{c|}{ SOLID WATER } \\
\cline { 2 - 5 } \multicolumn{1}{c|}{} & \multicolumn{4}{c|}{ 9 FIELDS PLAN } \\
\cline { 2 - 5 } & $\mathrm{C}$ & FSS & $\mathrm{S}$ & HB \\
\cline { 2 - 5 } & 1.01 & 1.01 & 1.02 & 1 \\
\cline { 2 - 5 } & 1.02 & 0.99 & 1.02 & 1.01 \\
\cline { 2 - 5 } & 1.01 & 1 & 1.01 & 1 \\
\cline { 2 - 5 } & 1.01 & 1.01 & 1.01 & 1.01 \\
\cline { 2 - 5 } & 1.01 & 1.01 & 1.01 & 1.01 \\
\cline { 2 - 5 } & 1.02 & 1.01 & 1.01 & 1.01 \\
\hline Average & 1.01 & 1.01 & 1.01 & 1.01 \\
\hline STD & 0.005 & 0.008 & 0.005 & 0.005 \\
\hline \%Dev & 1 & 1 & 1 & 1 \\
\hline
\end{tabular}

\begin{tabular}{|c|c|c|c|c|}
\cline { 2 - 5 } \multicolumn{1}{c|}{} & \multicolumn{4}{c|}{ SOLID WATER } \\
\cline { 2 - 5 } \multicolumn{1}{c|}{} & \multicolumn{4}{c|}{12 FIELDS PLAN } \\
\cline { 2 - 5 } & C & FSS & S & HB \\
\cline { 2 - 5 } & 1 & 1.01 & 1 & 1 \\
\cline { 2 - 5 } & 1 & 1.01 & 1 & 1.01 \\
\cline { 2 - 5 } & 1 & 1.01 & 1.01 & 1.01 \\
\cline { 2 - 5 } & 1.01 & 1.01 & 1 & 1 \\
\cline { 2 - 5 } & 1 & 1.01 & 1 & 1.01 \\
\cline { 2 - 5 } & 1 & 1.01 & 1 & 1.01 \\
\hline Average & 1 & 1.01 & 1 & 1.01 \\
\hline STD & 0.004 & 0 & 0.004 & 0.005 \\
\hline \%Dev & 0 & 1 & 1 & 1 \\
\hline
\end{tabular}


Table 2: Comparison of absorbed doses in bone for different field plans, showing percentage deviation from reference dose (1.00Gy)for (a) Single field (b) Wedged field (c) Oblique fields (d) Oppose fields (e) Three fields (f) four fields (g) 6 fields (h) 9 fields and (i) 12 fields for different algorithms

\begin{tabular}{|c|c|c|c|c|}
\hline \multirow{4}{*}{ (a) } & \multicolumn{4}{|c|}{ BONE } \\
\cline { 2 - 5 } & \multicolumn{4}{|c|}{ SINGLE FIELD } \\
\cline { 2 - 5 } & $\mathrm{C}$ & FSS & $\mathrm{S}$ & $\mathrm{HB}$ \\
\cline { 2 - 5 } & 1.04 & 1.02 & 1.02 & 1.01 \\
\cline { 2 - 5 } & 1.04 & 1.02 & 1.02 & 1 \\
\cline { 2 - 5 } & 1.04 & 1.02 & 1.02 & 1 \\
\cline { 2 - 5 } & 1.04 & 1.01 & 1.02 & 1 \\
\cline { 2 - 5 } & 1.03 & 1.01 & 1.02 & 1 \\
\cline { 2 - 5 } & 1.04 & 1.01 & 1.02 & 1 \\
\hline Average & 1.04 & 1.01 & 1.02 & 1 \\
\hline STD & 0.004 & 0.005 & 0 & 0.004 \\
\hline \%Dev & 4 & 1 & 2 & 0 \\
\hline
\end{tabular}

\begin{tabular}{|c|c|c|c|c|}
\cline { 2 - 5 }$(\mathrm{c})$ & \multicolumn{4}{|c|}{ BONE } \\
\cline { 2 - 5 } & \multicolumn{4}{|c|}{ WEDGE FIELDS } \\
\cline { 2 - 5 } & $\mathrm{C}$ & FSS & $\mathrm{S}$ & HB \\
\cline { 2 - 5 } & 1.04 & 1.02 & 1.03 & 1.02 \\
\cline { 2 - 5 } & 1.04 & 1.03 & 1.03 & 1.02 \\
\cline { 2 - 5 } & 1.04 & 1.03 & 1.03 & 1.01 \\
\cline { 2 - 5 } & 1.04 & 1.03 & 1.03 & 1.02 \\
\cline { 2 - 5 } & 1.04 & 1.03 & 1.03 & 1.02 \\
\cline { 2 - 5 } & 1.04 & 1.03 & 1.03 & 1 \\
\hline AVERAGE & 1.04 & 1.03 & 1.03 & 1.02 \\
\hline STD & 0 & 0.004 & 0 & 0.008 \\
\hline \%Dev & 4 & 3 & 3 & 2 \\
\hline
\end{tabular}

\begin{tabular}{|c|c|c|c|c|}
\hline \multirow{9}{*}{ (c) } & \multicolumn{4}{|c|}{ BONE } \\
\hline & \multicolumn{4}{|c|}{ OBLIQUE FIELDS } \\
\hline & $=$ & ss & 5 & $B$ \\
\hline & 03 & 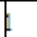 & 01 & 01 \\
\hline & 03 & 99 & 01 & \\
\hline & 04 & 01 & 02 & 01 \\
\hline & 04 & 01 & 02 & \\
\hline & 04 & 01 & 02 & 01 \\
\hline & 03 & 01 & 02 & 01 \\
\hline GE & 03 & 01 & 02 & 01 \\
\hline & 605 & bos & 05 & 05 \\
\hline & 5 & & 2 & \\
\hline
\end{tabular}

\begin{tabular}{|c|c|c|c|c|}
\hline \multirow{4}{*}{$(d)$} & \multicolumn{4}{|c|}{ BONE } \\
\cline { 2 - 5 } & \multicolumn{3}{|c|}{2 OPP FIELDS } \\
\cline { 2 - 5 } & $\mathrm{C}$ & FSS & $\mathrm{S}$ & HB \\
\cline { 2 - 5 } & 0.97 & 0.95 & 0.95 & 0.99 \\
\cline { 2 - 5 } & 0.99 & 0.97 & 0.97 & 0.98 \\
\cline { 2 - 5 } & 0.98 & 0.96 & 0.97 & 0.99 \\
\cline { 2 - 5 } & 0.98 & 0.96 & 0.97 & 0.99 \\
\cline { 2 - 5 } & 0.98 & 0.96 & 0.97 & 1 \\
\cline { 2 - 5 } & 0.98 & 0.96 & 0.97 & 0.99 \\
\hline AVERAGE & 0.98 & 0.96 & 0.97 & 0.99 \\
\hline STD & 0.006 & 0.006 & 0.008 & 0.006 \\
\hline \%Dev & -2 & -4 & -3 & -1 \\
\hline
\end{tabular}

\begin{tabular}{|l|c|c|c|c|}
\hline \multirow{4}{*}{ (e) } & \multicolumn{4}{|c|}{ BONE } \\
\cline { 2 - 5 } & \multicolumn{4}{|c|}{ THREE FIELDS } \\
\cline { 2 - 5 } & $\mathrm{C}$ & FSS & $\mathrm{S}$ & HB \\
\cline { 2 - 5 } & 1.03 & 1 & 1.01 & 1 \\
\cline { 2 - 5 } & 1.03 & 1.01 & 1.01 & 1.01 \\
\cline { 2 - 5 } & 1.03 & 1.01 & 1.02 & 1 \\
\cline { 2 - 5 } & 1.03 & 1.01 & 1.01 & 1 \\
\cline { 2 - 5 } & 1.02 & 1.01 & 1.01 & 1 \\
\cline { 2 - 5 } & 1.02 & 1 & 1.02 & 1 \\
\hline AVERAGE & 1.03 & 1.01 & 1.01 & 1 \\
\hline STD & 0.006 & 0.005 & 0.005 & 0.004 \\
\hline \%Dav & 3 & 1 & 1 & 0 \\
\hline
\end{tabular}

\begin{tabular}{|l|c|c|c|c|}
\hline \multirow{4}{*}{ (f) } & \multicolumn{4}{|c|}{ BONE } \\
\cline { 2 - 5 } & \multicolumn{3}{|c|}{ 4 FIELDS PLAN } \\
\cline { 2 - 5 } & $\mathrm{C}$ & FSS & $\mathrm{S}$ & HB \\
\cline { 2 - 5 } & 1.02 & 1.01 & 1.02 & 1.01 \\
\cline { 2 - 5 } & 1.02 & 1.02 & 1.02 & 1.01 \\
\cline { 2 - 5 } & 1.02 & 1.02 & 1.02 & 1.01 \\
\cline { 2 - 5 } & 1.02 & 1.01 & 1.02 & 1.01 \\
\cline { 2 - 5 } & 1.02 & 1.01 & 1.02 & 1 \\
\cline { 2 - 5 } & 1.02 & 1.02 & 1.02 & 1.01 \\
\hline Average & 1.02 & 1.01 & 1.02 & 1 \\
\hline STD & 0 & 0.005 & 0 & 0.005 \\
\hline \%Dev & 2 & 1 & 2 & 1 \\
\hline
\end{tabular}




\begin{tabular}{|c|c|c|c|c|}
\hline \multirow{4}{*}{$(\mathbf{n})$} & \multicolumn{4}{|c|}{ BONE } \\
\cline { 2 - 5 } & \multicolumn{3}{|c|}{6 FIELDS PLAN } \\
\cline { 2 - 5 } & C & FSS & S & HB \\
\cline { 2 - 5 } & 1.01 & 1.02 & 1.01 & 1 \\
\cline { 2 - 5 } & 1.02 & 1.01 & 1.01 & 1 \\
\cline { 2 - 5 } & 1.02 & 1.02 & 1.01 & 1.01 \\
\cline { 2 - 5 } & 1.02 & 1.02 & 1.01 & 1.01 \\
\cline { 2 - 5 } & 1.02 & 1.01 & 1.01 & 1.01 \\
\cline { 2 - 5 } & 1.02 & 1.02 & 1.01 & 1.01 \\
\hline AVERAGE & 1.02 & 1.02 & 1.01 & 1.01 \\
\hline STD & 0.004 & 0.005 & 0 & 0.005 \\
\hline \%Dev & 2 & 2 & 1 & 1 \\
\hline
\end{tabular}

\begin{tabular}{|l|c|c|c|c|}
\hline \multirow{4}{*}{ (h) } & \multicolumn{4}{|c|}{ BONE } \\
\cline { 2 - 5 } & \multicolumn{3}{|c|}{9 FIELDS PLAN } \\
\cline { 2 - 5 } & C & FSS & S & HB \\
\cline { 2 - 5 } & 1.01 & 1.01 & 1.02 & 1 \\
\cline { 2 - 5 } & 1.02 & 0.99 & 1.02 & 1.01 \\
\cline { 2 - 5 } & 1.01 & 1 & 1.01 & 1 \\
\cline { 2 - 5 } & 1.01 & 1.01 & 1.01 & 1.01 \\
\cline { 2 - 5 } & 1.01 & 1.01 & 1.01 & 1.01 \\
\cline { 2 - 5 } & 1.02 & 1.01 & 1.01 & 1.01 \\
\hline AVERAGE & 1.01 & 1.01 & 1.01 & 1.01 \\
\hline STD & 0.005 & 0.008 & 0.005 & 0.005 \\
\hline \%Dav & 1 & 1 & 1 & 1 \\
\hline
\end{tabular}

Table 3: Comparison of absorbed doses in lung for different field plans, showing percentage deviation from reference dose (1.00Gy)for (a) Single field (b) Wedged field (c) Oblique fields (d) Oppose fields (e) Three fields (f) four fields (g) 6 fields (h) 9 fields and (i) 12 fields for different algorithms

\begin{tabular}{|l|c|c|c|c|}
\hline \multirow{4}{*}{ (a) } & \multicolumn{4}{|c|}{ LUNG } \\
\cline { 2 - 5 } & \multicolumn{3}{|c|}{ SINGLE FIELD } \\
\cline { 2 - 5 } & C & FSS & S & HB \\
\cline { 2 - 5 } & 1.03 & 1.02 & 1.03 & 1 \\
\cline { 2 - 5 } & 1.02 & 1.01 & 1.02 & 1.01 \\
\cline { 2 - 5 } & 1.02 & 1.01 & 1.02 & 1.01 \\
\cline { 2 - 5 } & 1.02 & 1.01 & 1.02 & 1.01 \\
\cline { 2 - 5 } & 1.02 & 1.01 & 1.02 & 1 \\
\cline { 2 - 5 } & 1.02 & 1.01 & 1.02 & 1 \\
\hline AVERAGE & 1.02 & 1.01 & 1.02 & 1.01 \\
\hline STD & 0.004 & 0.004 & 0.004 & 0.005 \\
\hline \%Dav & 2 & 1 & 2 & 1 \\
\hline
\end{tabular}

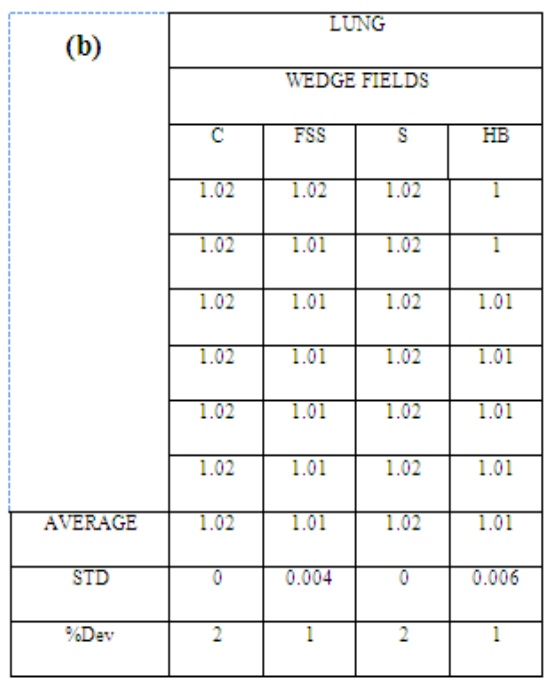

\begin{tabular}{|l|c|c|c|c|}
\hline \multirow{4}{*}{ (c) } & \multicolumn{4}{|c|}{ LUNG } \\
\cline { 2 - 5 } & \multicolumn{3}{|c|}{ OBLIQUE FIELDS } \\
\cline { 2 - 5 } & C & FSS & S & HB \\
\cline { 2 - 5 } & 1.03 & 1.01 & 1.03 & 1.01 \\
\cline { 2 - 5 } & 1.02 & 1.02 & 1.02 & 1.01 \\
\cline { 2 - 5 } & 1.02 & 1.01 & 1.02 & 1.01 \\
\cline { 2 - 5 } & 1.02 & 1.01 & 1.02 & 1.01 \\
\cline { 2 - 5 } & 1.02 & 1.01 & 1.02 & 1.01 \\
\cline { 2 - 5 } & 1.02 & 1.01 & 1.02 & 1.01 \\
\hline AVERAGE & 1.02 & 1.01 & 1.02 & 1.01 \\
\hline STD & 0.004 & 0.004 & 0.004 & 0 \\
\hline \%Dav & 2 & 1 & 2 & 1 \\
\hline
\end{tabular}

\begin{tabular}{|c|c|c|c|c|}
\hline \multirow{4}{*}{$(d)$} & \multicolumn{4}{|c|}{ LUNG } \\
\cline { 2 - 5 } & \multicolumn{4}{|c|}{ OPPOSING FIELDS } \\
\cline { 2 - 5 } & C & FSS & S & HB \\
\cline { 2 - 5 } & 0.98 & 0.97 & 0.97 & 0.99 \\
\cline { 2 - 5 } & 0.97 & 0.96 & 0.97 & 0.98 \\
\cline { 2 - 5 } & 0.97 & 0.97 & 0.97 & 0.99 \\
\cline { 2 - 5 } & 0.97 & 0.97 & 0.97 & 0.99 \\
\cline { 2 - 5 } & 0.97 & 0.97 & 0.97 & 0.99 \\
\cline { 2 - 5 } & 0.97 & 0.97 & 0.98 & 0.98 \\
\hline AVERAGE & 0.97 & 0.97 & 0.97 & 0.99 \\
\hline STD & 0.004 & 0.004 & 0.004 & 0.004 \\
\hline \%Dav & -3 & -3 & -3 & -1 \\
\hline
\end{tabular}




\begin{tabular}{|c|c|c|c|c|}
\hline \multirow{4}{*}{ (e) } & \multicolumn{4}{|c|}{ LUNG } \\
\cline { 2 - 5 } & \multicolumn{4}{|c|}{ THREE FIELDS } \\
\cline { 2 - 5 } & $\mathrm{C}$ & FSS & $\mathrm{S}$ & HB \\
\cline { 2 - 5 } & 1.02 & 1.01 & 1.01 & 1 \\
\cline { 2 - 5 } & 1.02 & 1.01 & 1.01 & 1.01 \\
\cline { 2 - 5 } & 1.01 & 0.99 & 1 & 1 \\
\cline { 2 - 5 } & 1.01 & 0.99 & 1 & 1 \\
\cline { 2 - 5 } & 1.01 & 0.99 & 1.01 & 1 \\
\cline { 2 - 5 } & 1.01 & 1 & 1.01 & 1 \\
\hline AVERAGE & 1.01 & 0.99 & 1.01 & 1 \\
\hline STD & 0.005 & 0.009 & 0.005 & 0.004 \\
\hline \%Dav & 1 & -1 & 1 & 0 \\
\hline
\end{tabular}

\begin{tabular}{|l|c|c|c|c|}
\hline \multirow{4}{*}{$(f)$} & \multicolumn{4}{|c|}{ LUNG } \\
\cline { 2 - 5 } & \multicolumn{3}{|c|}{ 4 FIELDS PLAN } \\
\cline { 2 - 5 } & $\mathrm{C}$ & FSS & $\mathrm{S}$ & HB \\
\cline { 2 - 5 } & 1.02 & 1.01 & 1.01 & 1.02 \\
\cline { 2 - 5 } & 1.01 & 1.01 & 1.02 & 1.01 \\
\cline { 2 - 5 } & 1.02 & 1.02 & 1.01 & 1.02 \\
\cline { 2 - 5 } & 1.02 & 1.02 & 1.02 & 1.02 \\
\cline { 2 - 5 } & 1.02 & 1.02 & 1.02 & 1.01 \\
\cline { 2 - 5 } & 1.02 & 1.02 & 1.02 & 1.02 \\
\hline AVERAGE & 1.02 & 1.02 & 1.02 & 1.02 \\
\hline STD & 0.004 & 0.005 & 0.005 & 0.005 \\
\hline \%Dsv & 2 & 2 & 2 & 2 \\
\hline
\end{tabular}

\begin{tabular}{|c|c|c|c|c|}
\hline \multirow{4}{*}{$(\mathbf{g})$} & \multicolumn{4}{|c|}{ LUNG } \\
\cline { 2 - 5 } & \multicolumn{3}{|c|}{6 FIELDS PLAN } \\
\cline { 2 - 5 } & $\mathrm{C}$ & FSS & S & HB \\
\cline { 2 - 5 } & 1.01 & 1.01 & 1.02 & 1.01 \\
\cline { 2 - 5 } & 1.02 & 1.02 & 1.01 & 1.01 \\
\cline { 2 - 5 } & 1.02 & 1.02 & 1.01 & 1 \\
\cline { 2 - 5 } & 1.02 & 1.02 & 1.01 & 1.01 \\
\cline { 2 - 5 } & 1.02 & 1.02 & 1.01 & 1.01 \\
\cline { 2 - 5 } & 1.02 & 1.02 & 1.01 & 1 \\
\hline AVERAGE & 1.02 & 1.02 & 1.01 & 1.01 \\
\hline STD & 0.004 & 0.004 & 0.004 & 0.005 \\
\hline \%Dav & 2 & 2 & 1 & 1 \\
\hline
\end{tabular}

\begin{tabular}{|l|c|c|c|c|}
\hline \multirow{4}{*}{$($ (h) } & \multicolumn{4}{|c|}{ LUNG } \\
\cline { 2 - 5 } & \multicolumn{4}{|c|}{9 FIELDS PLAN } \\
\cline { 2 - 5 } & C & FSS & S & HB \\
\cline { 2 - 5 } & 1.01 & 1.01 & 1.01 & 1 \\
\cline { 2 - 5 } & 1.01 & 1.01 & 1 & 1 \\
\cline { 2 - 5 } & 1.01 & 1.01 & 1 & 1 \\
\cline { 2 - 5 } & 1.01 & 1.01 & 1 & 1 \\
\cline { 2 - 5 } & 1.02 & 1.02 & 1 & 1 \\
\cline { 2 - 5 } & 1.02 & 1.01 & 1.01 & 1.01 \\
\hline AVERAGE & 1.01 & 1.01 & 1 & 1 \\
\hline STD & 0.005 & 0.004 & 0.005 & 0.004 \\
\hline \%Dev & 1 & 1 & 0 & 0 \\
\hline
\end{tabular}

\begin{tabular}{|c|c|c|c|c|}
\hline \multirow{4}{*}{ (i) } & \multicolumn{4}{|c|}{ LUNG } \\
\cline { 2 - 5 } & \multicolumn{4}{|c|}{12 FIELDS PLAN } \\
\cline { 2 - 5 } & C & FSS & S & HB \\
\cline { 2 - 5 } & 1.01 & 1.02 & 1 & 1.01 \\
\cline { 2 - 5 } & 1.01 & 1.01 & 1 & 1 \\
\cline { 2 - 5 } & 1.01 & 1.01 & 1 & 1 \\
\cline { 2 - 5 } & 1.01 & 1.01 & 1 & 1 \\
\cline { 2 - 5 } & 1.01 & 1.01 & 1.01 & 1 \\
\hline AVERAGE & 1.01 & 1.01 & 1 & 1 \\
\hline STD & 0.004 & 0.004 & 0.004 & 0.004 \\
\hline \%Dav & 1 & 1 & 0 & 0 \\
\hline
\end{tabular}




\section{Results of the calculated times of the different algorithms for different plans}

Table 4 shows the results of the times used by the different algorithms for calculation of monitor units needed to deliver the prescribed dose for different plans.

Table 4: Calculation time of the different algorithms for different plans

\begin{tabular}{|l|c|c|c|}
\hline CASE & Hybrid (s) & Convolution (s) & Superposition (s) \\
\hline Lung (single field) & 3 & 0.5 & 1 \\
\hline Lung (Opposite fields) & 3 & 0.7 & 1.2 \\
\hline Lung (12 fields) & 4 & 7 & 8 \\
\hline Bone (12 fields) & 6 & 9 & 11 \\
\hline Solid water (12 fields) & 5 & 6 & 8 \\
\hline Lung (IMRT plan, 30 fields) & 12 & 45 & 66 \\
\hline Lungs (Rapid arc plan, 57fields) & 20 & 98 & 123 \\
\hline
\end{tabular}

Absorbed dose measured at the LINAC for $18 \mathrm{MeV}$ photon beam using the different algorithms

Table 5: Comparison of absorbed doses in solid water phantom for (a) Single field (b) Wedged field (c) Oblique fields (d) Oppose fields (e) Three fields (f) 4 fields (g) 6 fields (h) 9 fields and (i) 12 fields with different algorithms

\begin{tabular}{|l|c|c|c|c|}
\hline \multirow{4}{*}{ (a) } & \multicolumn{4}{|c|}{ SOLID WATER } \\
\cline { 2 - 5 } & \multicolumn{4}{|c|}{ SINGLE FIELD } \\
\cline { 2 - 5 } & $\mathrm{C}$ & FSS & $\mathrm{S}$ & HB \\
\cline { 2 - 5 } & 1.01 & 1.02 & 1.01 & 1.02 \\
\cline { 2 - 5 } & 1.02 & 1.01 & 1.02 & 1.01 \\
\cline { 2 - 5 } & 1.02 & 1.02 & 1.03 & 1.02 \\
\cline { 2 - 5 } & 1.01 & 1.01 & 1.02 & 1.02 \\
\cline { 2 - 5 } & 1.02 & 1.01 & 1.02 & 1.02 \\
\cline { 2 - 5 } & 1.01 & 1.01 & 1.03 & 1.02 \\
\hline Averagse & 1.02 & 1.01 & 1.02 & 1.02 \\
\hline STD & 0.006 & 0.005 & 0.008 & 0.004 \\
\hline \%Dav & 2 & 1 & 2 & 2 \\
\hline
\end{tabular}

\begin{tabular}{|l|c|c|c|c|}
\hline \multirow{4}{*}{ (c) } & \multicolumn{4}{|c|}{ SOLID WATER } \\
\cline { 2 - 5 } & \multicolumn{4}{|c|}{ OBLIQUE FIELDS } \\
\cline { 2 - 5 } & $\mathrm{C}$ & FSS & $\mathrm{S}$ & HB \\
\cline { 2 - 5 } & 1.01 & 1.01 & 1.01 & 1.01 \\
\cline { 2 - 5 } & 1.01 & 1.01 & 1.02 & 1.02 \\
\cline { 2 - 5 } & 1.01 & 1.01 & 1.02 & 1.02 \\
\cline { 2 - 5 } & 1.01 & 1.02 & 1.02 & 1.02 \\
\cline { 2 - 5 } & 1.01 & 1.02 & 1.01 & 1.02 \\
\cline { 2 - 5 } & 1.01 & 1.01 & 1.02 & 1.01 \\
\hline Avarage & 1.01 & 1.01 & 1.02 & 2 \\
\hline STD & 0.000 & 0.005 & 0.005 & 0.005 \\
\hline \%Dev & 1 & 1 & 2 & 2 \\
\hline
\end{tabular}

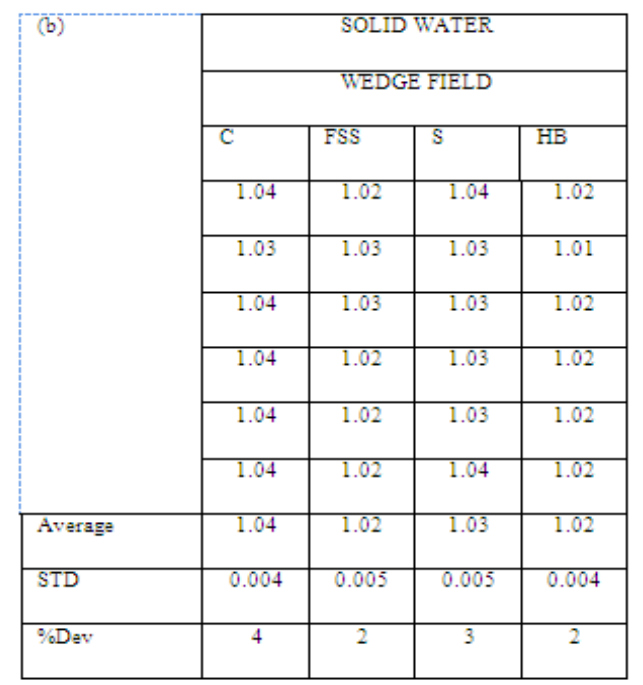

\begin{tabular}{|l|c|c|c|c|}
\hline \multirow{4}{*}{ (d) } & \multicolumn{4}{|c|}{ SOLID WATER } \\
\cline { 2 - 5 } & \multicolumn{3}{|c|}{ OPOSSING FIELDS } \\
\cline { 2 - 5 } & $\mathrm{C}$ & FSS & $\mathrm{S}$ & HB \\
\cline { 2 - 5 } & 1.03 & 1.02 & 1.01 & 1.02 \\
\cline { 2 - 5 } & 1.02 & 1.01 & 1.02 & 1.01 \\
\cline { 2 - 5 } & 1.02 & 1.01 & 1.02 & 1.01 \\
\cline { 2 - 5 } & 1.02 & 1.02 & 1.02 & 1.01 \\
\cline { 2 - 5 } & 1.02 & 1.01 & 1.02 & 1.01 \\
\cline { 2 - 5 } & 1.02 & 1.01 & 1.02 & 1.01 \\
\hline Averags & 1.02 & 1.01 & 1.02 & 1.01 \\
\hline STD & 0.004 & 0.005 & 0.004 & 0.004 \\
\hline \%Dav & 2 & 1 & 2 & 1 \\
\hline
\end{tabular}


Hybrid Algorithm For Dose Calculation In Cms Xio Treatment Planning System

\begin{tabular}{|l|c|c|c|c|}
\hline \multirow{4}{*}{ (e) } & \multicolumn{4}{|c|}{ SOLID WATER } \\
\cline { 2 - 5 } & \multicolumn{4}{|c|}{ THREE FIELDS } \\
\cline { 2 - 5 } & C & FSS & S & HB \\
\cline { 2 - 5 } & 1.02 & 1.01 & 1.02 & 1.01 \\
\cline { 2 - 5 } & 1.01 & 1.01 & 1.02 & 1.01 \\
\cline { 2 - 5 } & 1.02 & 1 & 1.01 & 1.02 \\
\cline { 2 - 5 } & 1.02 & 1.01 & 1.02 & 1.02 \\
\cline { 2 - 5 } & 1.01 & 1 & 1.01 & 1.02 \\
\cline { 2 - 5 } & 1.02 & 1.01 & 1.02 & 1.02 \\
\hline Averags & 1.02 & 1.01 & 1.02 & 1.02 \\
\hline STD & 0.005 & 0.005 & 0.005 & 0.005 \\
\hline \%Dev & 2 & 1 & 2 & 2 \\
\hline
\end{tabular}

\begin{tabular}{|l|c|c|c|c|}
\hline \multirow{4}{*}{$(\mathrm{f})$} & \multicolumn{4}{|c|}{ SOLID WATER } \\
\cline { 2 - 5 } & \multicolumn{4}{|c|}{ 4 FIELDS PLAN } \\
\cline { 2 - 5 } & $\mathrm{C}$ & FSS & $\mathrm{S}$ & $\mathrm{HB}$ \\
\cline { 2 - 5 } & 1.01 & 1.02 & 1.01 & 1.02 \\
\cline { 2 - 5 } & 1.02 & 1.01 & 1.01 & 1.03 \\
\cline { 2 - 5 } & 1.01 & 1.01 & 1.01 & 1.01 \\
\cline { 2 - 5 } & 1.01 & 1.01 & 1.02 & 1.02 \\
\cline { 2 - 5 } & 1.01 & 1.01 & 1.02 & 1.02 \\
\cline { 2 - 5 } & 1.01 & 1.01 & 1.02 & 1.01 \\
\hline Averags & 1.01 & 1.01 & 1.02 & 1.02 \\
\hline STD & 0.004 & 0.004 & 0.006 & 0.008 \\
\hline \%Dav & 1 & 1 & 2 & 2 \\
\hline
\end{tabular}
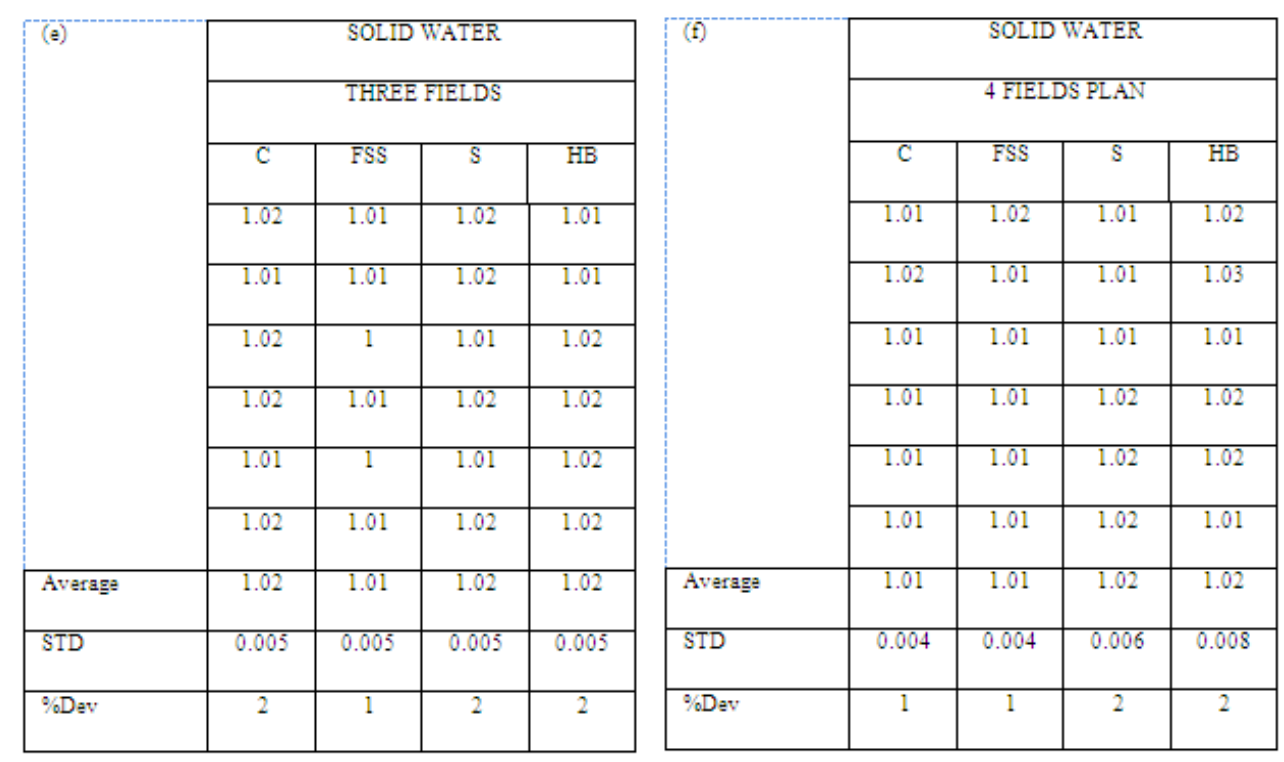

\begin{tabular}{|l|c|c|c|c|}
\hline \multirow{4}{*}{$(\mathrm{g})$} & \multicolumn{4}{|c|}{ SOLID WATER } \\
\cline { 2 - 5 } & \multicolumn{4}{|c|}{ 6 FIELDS PLAN } \\
\cline { 2 - 5 } & $\mathrm{C}$ & FSS & $\mathrm{S}$ & HB \\
\cline { 2 - 5 } & 1.01 & 1.02 & 1.02 & 1.02 \\
\cline { 2 - 5 } & 1.01 & 1.02 & 1.01 & 1.02 \\
\cline { 2 - 5 } & 1.01 & 1.02 & 1.02 & 1.02 \\
\cline { 2 - 5 } & 1.01 & 1.02 & 1.02 & 1.02 \\
\cline { 2 - 5 } & 1.01 & 1.01 & 1.02 & 1.01 \\
\cline { 2 - 5 } & 1.01 & 1.02 & 1.02 & 1.01 \\
\hline Average & 1.01 & 1.02 & 1.02 & 1.02 \\
\hline STD & 0.000 & 0.004 & 0.004 & 0.005 \\
\hline \%Dav & 1 & 2 & 2 & 2 \\
\hline
\end{tabular}

\begin{tabular}{|l|c|c|c|c|}
\hline \multirow{4}{*}{ (h) } & \multicolumn{4}{|c|}{ SOLID WATER } \\
\cline { 2 - 5 } & \multicolumn{4}{|c|}{9 FIELDS PLAN } \\
\cline { 2 - 5 } & $\mathrm{C}$ & FSS & $\mathrm{S}$ & HB \\
\cline { 2 - 5 } & 1 & 1.02 & 1.01 & 1.01 \\
\cline { 2 - 5 } & 1 & 1.03 & 1.01 & 1.02 \\
\cline { 2 - 5 } & 1 & 1.01 & 1.02 & 1.01 \\
\cline { 2 - 5 } & 1.01 & 1.02 & 1.01 & 1.01 \\
\cline { 2 - 5 } & 1.01 & 1.02 & 1.02 & 1.01 \\
\hline & 1 & 1.02 & 1.02 & 1.01 \\
\hline Averags & 1.00 & 1.02 & 1.02 & 1.01 \\
\hline STD & 0.005 & 0.008 & 0.006 & 0.004 \\
\hline \%Dav & 0 & 2 & 2 & 1 \\
\hline
\end{tabular}




\begin{tabular}{|l|c|c|c|c|}
\hline \multirow{4}{*}{} & \multicolumn{4}{|c|}{ SOLID WATER } \\
\cline { 2 - 5 } & \multicolumn{4}{|c|}{12 FIELDS PLAN } \\
\cline { 2 - 5 } & C & FSS & S & HB \\
\cline { 2 - 5 } & 1.01 & 1.02 & 1.01 & 1 \\
\cline { 2 - 5 } & 1.01 & 1.02 & 1.01 & 1.02 \\
\cline { 2 - 5 } & 1.01 & 1.01 & 1.01 & 1.01 \\
\cline { 2 - 5 } & 1.02 & 1.02 & 1.01 & 1.01 \\
\cline { 2 - 5 } & 1.01 & 1.02 & 1.01 & 1.02 \\
\cline { 2 - 5 } & 1.02 & 1.03 & 1.01 & 1.01 \\
\hline Average & 1 & 1.02 & 1.01 & 1.01 \\
\hline STD & 0.005 & 0.008 & 0 & 0.008 \\
\hline \%Dev & 1 & 2 & 1 & 1 \\
\hline
\end{tabular}

Table 6: Comparison of absorbed dose in bone for different field plans showing percentage deviation from reference dose (1.00Gy)for (a) Single field (b) Wedged field (c) Oblique fields (d) Oppose fields (e) Three field (f) 4 fields (g) 6 fields (h) 9 fields and (i) 12 fields with different algorithms.

\begin{tabular}{|l|c|c|c|c|}
\multirow{4}{*}{} & \multicolumn{4}{|c|}{ (a) } \\
\cline { 2 - 5 } & \multicolumn{3}{|c|}{ SINGLE FIELD } \\
\cline { 2 - 5 } & C & FSS & S & HB \\
\cline { 2 - 5 } & 1.03 & 1.03 & 1.01 & 1.01 \\
\cline { 2 - 5 } & 1.01 & 1.03 & 1.02 & 1.02 \\
\cline { 2 - 5 } & 1.03 & 1.02 & 1.01 & 1.02 \\
\cline { 2 - 5 } & 1.02 & 1.02 & 1.01 & 1.02 \\
\cline { 2 - 5 } & 1.03 & 1.02 & 1.01 & 1.02 \\
\cline { 2 - 5 } & 1.03 & 1.02 & 1.01 & 1.02 \\
\hline Average & 1.03 & 1.02 & 1.01 & 1.02 \\
\hline STD & 0.008 & 0.005 & 0.004 & 0.004 \\
\hline \%Dev & 3 & 2 & 1 & 2 \\
\hline
\end{tabular}

\begin{tabular}{|l|c|c|c|c|}
\hline \multirow{4}{*}{ (c) } & \multicolumn{4}{|c|}{ BONE } \\
\cline { 2 - 5 } & \multicolumn{3}{|c|}{ OBLIQUE FIELDS } \\
\cline { 2 - 5 } & C & FSS & S & HB \\
\cline { 2 - 5 } & 1.05 & 1.02 & 1.02 & 1.02 \\
\cline { 2 - 5 } & 1.03 & 1.02 & 1.01 & 1.02 \\
\cline { 2 - 5 } & 1.04 & 1.02 & 1.01 & 1.02 \\
\cline { 2 - 5 } & 1.03 & 1.03 & 1.01 & 1.01 \\
\cline { 2 - 5 } & 1.04 & 1.03 & 1.01 & 1.02 \\
\cline { 2 - 5 } & 1.03 & 1.02 & 1.01 & 1.02 \\
\hline AVERAGE & 1.04 & 1.02 & 1.01 & 1.02 \\
\hline STD & 0.006 & 0.005 & 0.004 & 0.004 \\
\hline \%Dav & 4 & 2 & 1 & 2 \\
\hline
\end{tabular}

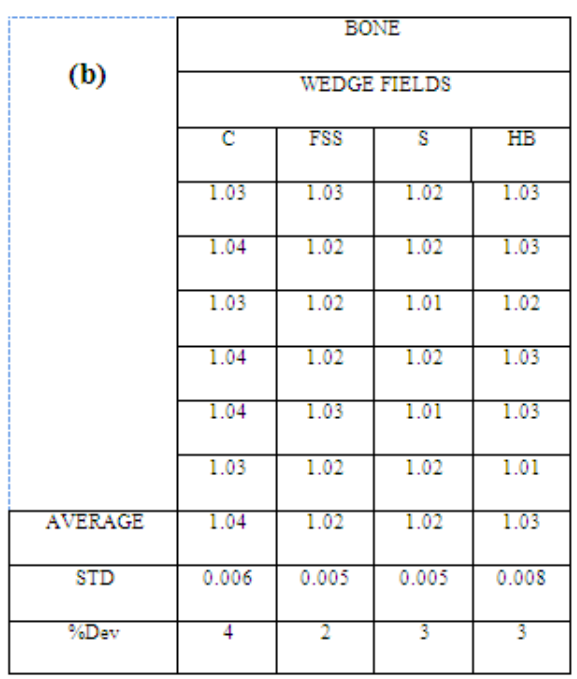

\begin{tabular}{|c|c|c|c|c|}
\hline \multirow{4}{*}{$(d)$} & \multicolumn{4}{|c|}{ BONE } \\
\cline { 2 - 5 } & \multicolumn{4}{|c|}{2 OPP FIELDS } \\
\cline { 2 - 5 } & C & FSS & S & HB \\
\cline { 2 - 5 } & 1.03 & 1.03 & 1.02 & 1.01 \\
\cline { 2 - 5 } & 1.03 & 1.02 & 1.02 & 1.02 \\
\cline { 2 - 5 } & 1.03 & 1.02 & 1.02 & 1.01 \\
\cline { 2 - 5 } & 1.02 & 1.01 & 1.02 & 1.02 \\
\cline { 2 - 5 } & 1.03 & 1.02 & 1.02 & 1.02 \\
\cline { 2 - 5 } & 1.03 & 1.02 & 1.01 & 1.02 \\
\hline AVERAGE & 1.03 & 1.02 & 1.02 & 1.02 \\
\hline STD & 0.004 & 0.006 & 0.004 & 0.005 \\
\hline \%Dav & 3 & 2 & 2 & 2 \\
\hline
\end{tabular}


Hybrid Algorithm For Dose Calculation In Cms Xio Treatment Planning System

\begin{tabular}{|l|c|c|c|c|}
\hline \multirow{4}{*}{ (e) } & \multicolumn{4}{|c|}{ BONE } \\
\cline { 2 - 5 } & \multicolumn{4}{|c|}{ THREE FIELDS } \\
\cline { 2 - 5 } & $\mathrm{C}$ & FSS & $\mathrm{S}$ & HB \\
\cline { 2 - 5 } & 1.02 & 1.02 & 1.02 & 1.01 \\
\cline { 2 - 5 } & 1.03 & 1.01 & 1.02 & 1.01 \\
\cline { 2 - 5 } & 1.04 & 1.02 & 1.01 & 1.02 \\
\cline { 2 - 5 } & 1.04 & 1.02 & 1.01 & 1.01 \\
\cline { 2 - 5 } & 1.04 & 1.02 & 1.01 & 1.02 \\
\cline { 2 - 5 } & 1.03 & 1.01 & 1.01 & 1.01 \\
\hline AVERAGE & 1.04 & 1.02 & 1.01 & 1.01 \\
\hline STD & 0.006 & 0.005 & 0.005 & 0.005 \\
\hline \%Dav & 4 & 2 & 1 & 1 \\
\hline
\end{tabular}

\begin{tabular}{|l|c|c|c|c|}
\hline \multirow{4}{*}{$(f)$} & \multicolumn{4}{|c|}{ BONE } \\
\cline { 2 - 5 } & \multicolumn{4}{|c|}{4 FIELDS PLAN } \\
\cline { 2 - 5 } & $\mathrm{C}$ & FSS & $\mathrm{S}$ & $\mathrm{HB}$ \\
\cline { 2 - 5 } & 1.03 & 1.02 & 1.01 & 1.02 \\
\cline { 2 - 5 } & 1.03 & 1.02 & 1.01 & 1.02 \\
\cline { 2 - 5 } & 1.02 & 1.01 & 1.01 & 1.03 \\
\cline { 2 - 5 } & 1.02 & 1.02 & 1.02 & 1.02 \\
\cline { 2 - 5 } & 1.03 & 1.02 & 1.01 & 1.02 \\
\cline { 2 - 5 } & 1.03 & 1.02 & 1.01 & 1.01 \\
\hline Averags & 1.03 & 1.02 & 1.01 & 1.02 \\
\hline STD & 0.005 & 0.004 & 0.004 & 0.006 \\
\hline \%Dav & 3 & 2 & 1 & 2 \\
\hline
\end{tabular}

\begin{tabular}{|c|c|c|c|c|}
\hline \multirow{4}{*}{$(g)$} & \multicolumn{4}{|c|}{ BONE } \\
\cline { 2 - 5 } & \multicolumn{4}{|c|}{ 6 FIELDS PLAN } \\
\cline { 2 - 5 } & C & FSS & S & HB \\
\cline { 2 - 5 } & 1.03 & 1.01 & 1.02 & 1.01 \\
\cline { 2 - 5 } & 1.03 & 1.02 & 1.01 & 1.02 \\
\cline { 2 - 5 } & 1.02 & 1.01 & 1.01 & 1.01 \\
\cline { 2 - 5 } & 1.02 & 1.01 & 1.02 & 1.01 \\
\cline { 2 - 5 } & 1.02 & 1.01 & 1.01 & 1.02 \\
\cline { 2 - 5 } & 1.02 & 1.01 & 1.01 & 1.02 \\
\hline AVERAGE & 1.02 & 1.01 & 1.01 & 1.02 \\
\hline STD & 0.005 & 0.004 & 0.005 & 0.006 \\
\hline \%Dav & 2 & 1 & 1 & 2 \\
\hline
\end{tabular}

\begin{tabular}{|c|c|c|c|c|}
\hline \multirow{4}{*}{ (h) } & \multicolumn{4}{|c|}{ BONE } \\
\cline { 2 - 5 } & \multicolumn{4}{|c|}{9 FIELDS PLAN } \\
\cline { 2 - 5 } & C & FSS & S & HB \\
\cline { 2 - 5 } & 1.02 & 1.02 & 1 & 1.01 \\
\cline { 2 - 5 } & 1.01 & 1.02 & 1 & 1.01 \\
\cline { 2 - 5 } & 1.02 & 1.02 & 1.01 & 1.01 \\
\cline { 2 - 5 } & 1.02 & 1.02 & 1.01 & 1.01 \\
\cline { 2 - 5 } & 1.02 & 1.02 & 1 & 1.01 \\
\cline { 2 - 5 } & 1.01 & 1.01 & 1.01 & 1.01 \\
\hline AVERAGE & 1.02 & 1.02 & 1.01 & 1.01 \\
\hline STD & 0.005 & 0.004 & 0.006 & 0 \\
\hline \%Dav & 2 & 2 & 1 & 1 \\
\hline
\end{tabular}

\begin{tabular}{|l|c|c|c|c|}
\hline \multirow{4}{*}{ (i) } & \multicolumn{4}{|c|}{ BONE } \\
\cline { 2 - 5 } & \multicolumn{4}{|c|}{12 FIELDS } \\
\cline { 2 - 5 } & C & FSS & S & HB \\
\cline { 2 - 5 } & 1.02 & 1.01 & 1.01 & 1.01 \\
\cline { 2 - 5 } & 1.02 & 1.01 & 1.01 & 1.02 \\
\cline { 2 - 5 } & 1.03 & 1.01 & 1 & 1.01 \\
\cline { 2 - 5 } & 1.02 & 1.01 & 1.01 & 1.01 \\
\cline { 2 - 5 } & 1.02 & 1.01 & 1 & 1.01 \\
\cline { 2 - 5 } & 1.02 & 1.01 & 1.01 & 1.01 \\
\hline Average & 1.02 & 1.01 & 1.01 & 1.01 \\
\hline STD & 0.004 & 0 & 0.005 & 0.004 \\
\hline \%Dev & 2 & 1 & 1 & 1 \\
\hline
\end{tabular}


Table 7: Comparison of absorbed dose in lung for different field plans showing percentage deviation from reference dose (1.00Gy)for (a) Single field (b) Wedged field (c) Oblique fields (d) Oppose fields (e) Three field (f) 4 fields (g) 6 fields (h) 9 fields and (i) 12 fields with different algorithms.

\begin{tabular}{|l|c|c|c|c|}
\hline \multirow{4}{*}{ (a) } & \multicolumn{4}{|c|}{ LUNG } \\
\cline { 2 - 5 } & \multicolumn{4}{|c|}{ SINGLE FIELD } \\
\cline { 2 - 5 } & C & FSS & S & HB \\
\cline { 2 - 5 } & 1.02 & 1.01 & 1.01 & 1.02 \\
\cline { 2 - 5 } & 1.03 & 1.02 & 1.01 & 1.02 \\
\cline { 2 - 5 } & 1.02 & 1.02 & 1.02 & 1.02 \\
\cline { 2 - 5 } & 1.03 & 1.02 & 1.01 & 1.02 \\
\cline { 2 - 5 } & 1.03 & 1.02 & 1.01 & 1.01 \\
\cline { 2 - 5 } & 1.03 & 1.01 & 1.01 & 1.01 \\
\hline AVERAGE & 1.03 & 1.02 & 1.01 & 1.02 \\
\hline STD & 0.005 & 0.005 & 0.004 & 0.005 \\
\hline \%Dev & 3 & 2 & 1 & 2 \\
\hline
\end{tabular}

\begin{tabular}{|c|c|c|c|c|}
\hline \multirow{4}{*}{$(b)$} & \multicolumn{4}{|c|}{ LUNG } \\
\cline { 2 - 5 } & \multicolumn{4}{|c|}{ WEDGE FIELDS } \\
\cline { 2 - 5 } & C & FSS & S & HB \\
\cline { 2 - 5 } & 1.04 & 1.03 & 1.03 & 1.02 \\
\cline { 2 - 5 } & 1.03 & 1.03 & 1.03 & 1.03 \\
\cline { 2 - 5 } & 1.04 & 1.02 & 1.03 & 1.02 \\
\cline { 2 - 5 } & 1.04 & 1.02 & 1.03 & 1.02 \\
\cline { 2 - 5 } & 1.04 & 1.03 & 1.02 & 1.02 \\
\cline { 2 - 5 } & 1.04 & 1.03 & 1.03 & 1.02 \\
\hline AVERAGE & 1.04 & 1.03 & 1.03 & 1.02 \\
\hline STD & 0.004 & 0.005 & 0.004 & 0.004 \\
\hline \%Dev & 4 & 3 & 3 & 2 \\
\hline
\end{tabular}

\begin{tabular}{|l|c|c|c|c|}
\hline \multirow{4}{*}{} & \multicolumn{4}{|c|}{ LUNG } \\
\cline { 2 - 5 } & \multicolumn{3}{|c|}{ OBLIQUE FIELDS } \\
\cline { 2 - 5 } & C & FSS & S & HB \\
\cline { 2 - 5 } & 1.02 & 1.02 & 1.01 & 1.02 \\
\cline { 2 - 5 } & 1.03 & 1.02 & 1.01 & 1.02 \\
\cline { 2 - 5 } & 1.03 & 1.02 & 1.02 & 1.01 \\
\cline { 2 - 5 } & 1.03 & 1.02 & 1.01 & 1.02 \\
\cline { 2 - 5 } & 1.03 & 1.02 & 1.02 & 1.02 \\
\cline { 2 - 5 } & 1.02 & 1.02 & 1.01 & 1.02 \\
\hline AVERAGE & 1.03 & 1.02 & 1.01 & 1.02 \\
\hline STD & 0.005 & 0 & 0.005 & 0.004 \\
\hline \%Dev & 3 & 2 & 1 & 2 \\
\hline
\end{tabular}

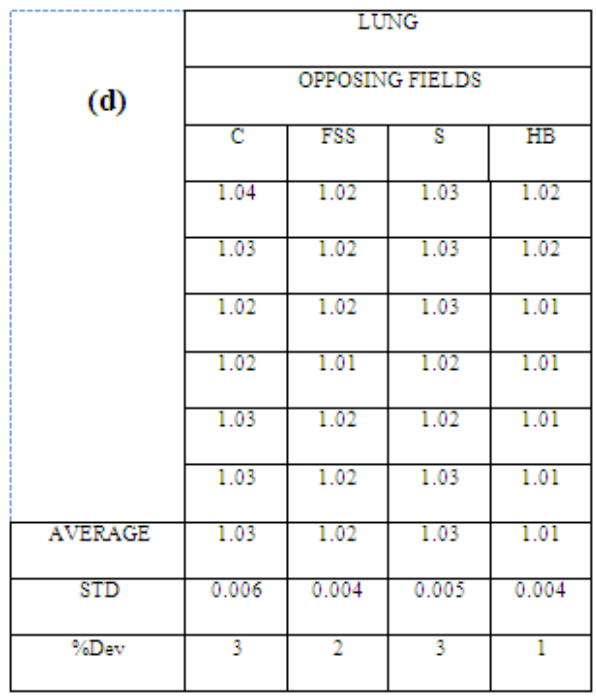

\begin{tabular}{|l|c|c|c|c|}
\hline \multirow{4}{*}{$(e)$} & \multicolumn{4}{|c|}{ LUNG } \\
\cline { 2 - 5 } & \multicolumn{4}{|c|}{ THREE FIELDS } \\
\cline { 2 - 5 } & C & FSS & S & HB \\
\cline { 2 - 5 } & 1.02 & 1.02 & 1.02 & 1.01 \\
\cline { 2 - 5 } & 1.02 & 1.01 & 1.01 & 1.01 \\
\cline { 2 - 5 } & 1.02 & 1.01 & 1.01 & 1.02 \\
\cline { 2 - 5 } & 1.02 & 1 & 1.01 & 1.02 \\
\cline { 2 - 5 } & 1.02 & 1.02 & 1.02 & 1.02 \\
\cline { 2 - 5 } & 1.01 & 1 & 1.02 & 1.02 \\
\hline AVERAGE & 1.02 & 1.01 & 1.02 & 1.02 \\
\hline STD & 0.004 & 0.006 & 0.006 & 0.005 \\
\hline \%Dav & 2 & 1 & 2 & 2 \\
\hline
\end{tabular}

\begin{tabular}{|c|c|c|c|c|}
\hline \multirow{4}{*}{$(f)$} & \multicolumn{4}{|c|}{ LUNG } \\
\cline { 2 - 5 } & \multicolumn{3}{|c|}{4 FIELDS PLAN } \\
\cline { 2 - 5 } & C & FSS & S & HB \\
\cline { 2 - 5 } & 1.03 & 1.02 & 1.02 & 1.01 \\
\cline { 2 - 5 } & 1.02 & 1.02 & 1.02 & 1.01 \\
\cline { 2 - 5 } & 1.03 & 1.01 & 1.02 & 1.01 \\
\cline { 2 - 5 } & 1.03 & 1.01 & 1.01 & 1.01 \\
\cline { 2 - 5 } & 1.03 & 1.01 & 1.02 & 1.01 \\
\cline { 2 - 5 } & 1.03 & 1.01 & 1.02 & 1.01 \\
\hline AVERAGE & 1.03 & 1.01 & 1.02 & 1.01 \\
\hline STD & 0.004 & 0.005 & 0.004 & 0 \\
\hline \%Dev & 3 & 1 & 2 & 1 \\
\hline
\end{tabular}




\begin{tabular}{|c|c|c|c|c|}
\hline \multirow{4}{*}{$(\mathbf{v})$} & \multicolumn{4}{|c|}{ LUNG } \\
\cline { 2 - 5 } & \multicolumn{4}{|c|}{6 FIELDS PLAN } \\
\cline { 2 - 5 } & C & FSS & S & HB \\
\cline { 2 - 5 } & 1.03 & 1.03 & 1.02 & 1.01 \\
\cline { 2 - 5 } & 1.03 & 1.02 & 1.02 & 1.01 \\
\cline { 2 - 5 } & 1.03 & 1.03 & 1.01 & 1.01 \\
\cline { 2 - 5 } & 1.02 & 1.03 & 1.01 & 1.02 \\
\cline { 2 - 5 } & 1.02 & 1.03 & 1.02 & 1.02 \\
\cline { 2 - 5 } & 1.03 & 1.03 & 1.02 & 1.02 \\
\hline AVERAGE & 1.03 & 1.03 & 1.02 & 1.02 \\
\hline STD & 0.005 & 0.004 & 0.005 & 0.006 \\
\hline \%Dav & 3 & 3 & 2 & 2 \\
\hline
\end{tabular}

\begin{tabular}{|l|c|c|c|c|}
\hline \multirow{4}{*}{} & \multicolumn{4}{|c|}{ LUNG } \\
\cline { 2 - 5 } & \multicolumn{3}{|c|}{9 FIELDS PLAN } \\
\cline { 2 - 5 } & C & FSS & S & HB \\
\cline { 2 - 5 } & 1.02 & 1.02 & 1.01 & 1.02 \\
\cline { 2 - 5 } & 1.02 & 1.01 & 1.01 & 1.02 \\
\cline { 2 - 5 } & 1.01 & 1.01 & 1.02 & 1.02 \\
\cline { 2 - 5 } & 1.02 & 1.01 & 1.01 & 1.02 \\
\cline { 2 - 5 } & 1.01 & 1.02 & 1.01 & 1.01 \\
\cline { 2 - 5 } & 1.02 & 1.01 & 1.01 & 1.01 \\
\hline AVERAGE & 1.02 & 1.01 & 1.01 & 1.02 \\
\hline STD & 0.005 & 0.005 & 0.004 & 0.005 \\
\hline \%Dev & 2 & 1 & 1 & 2 \\
\hline
\end{tabular}

\begin{tabular}{|c|c|c|c|c|}
\hline \multirow{4}{*}{$(\mathrm{i})$} & \multicolumn{4}{|c|}{ LUNG } \\
\cline { 2 - 5 } & \multicolumn{4}{|c|}{12 FIELDS PLAN } \\
\cline { 2 - 5 } & C & FSS & S & HB \\
\cline { 2 - 5 } & 1.02 & 1.01 & 1.02 & 1.01 \\
\cline { 2 - 5 } & 1.02 & 1.01 & 1.01 & 1.01 \\
\cline { 2 - 5 } & 1.01 & 1.01 & 1.01 & 1.02 \\
\cline { 2 - 5 } & 1.02 & 1.01 & 1.02 & 1.01 \\
\cline { 2 - 5 } & 1.02 & 1.01 & 1.01 & 1.01 \\
\cline { 2 - 5 } & 1.01 & 1.01 & 1.01 & 1.01 \\
\hline AVERAGE & 1.02 & 1.01 & 1.01 & 1.01 \\
\hline STD & 0.005 & 0.004 & 0.005 & 0.004 \\
\hline & & & 1 & 1 \\
\hline & 2 & 1 & 1 & \\
\hline & & & & \\
\hline & & & & \\
\hline
\end{tabular}

Results of the calculated times of the different algorithms for different plans for the $18 \mathrm{MeV}$ photon beam.

Table 8 shows the results of the times used by the different algorithms for calculation of monitor units needed to deliver the prescribed dose for different plan cases.

Table 8: calculation time of the different algorithms for different plans for $18 \mathrm{MeV}$ photon beam

\begin{tabular}{|l|c|c|c|}
\hline CASE & Hybrid (s) & Convolution (s) & Superposition (s) \\
\hline Lung (single field) & 5 & 1.2 & 2 \\
\hline Lung (Opposite fields) & 6 & 1.8 & 3 \\
\hline Lung (12 fields) & 8 & 12 & 15 \\
\hline Bone (12 fields) & 9 & 13 & 18 \\
\hline Solid water (12 fields) & 7 & 10 & 14 \\
\hline Lung (IMRT plan, 30 fields) & 18 & 80 & 102 \\
\hline Lungs (Rapid arc plan, 57fields) & 27 & 135 & 186 \\
\hline
\end{tabular}




\section{Discussion and conclusion}

Table 1 shows the result of the absorbed dose measured in solid water along with the percentage deviation from the reference dose $(1.00 \mathrm{~Gy})$ and the standard deviation between the 6 measurements taken for the $6 \mathrm{MV}$ photon beam. FSS and HB showed better accuracy (1\%dev.) in tables 1 (a), (b) and (f). The C, S and HB algorithms were better ( $1 \%$ dev.) for the oblique and opposed fields as shown in tables 1 (c) and (d) while FSS, S and HB showed improved accuracy ( $1 \%$ dev.) for the 3 field plans in table 1 (e). The $\mathrm{C}$ algorithm had better accuracy (0\% dev.) in the 6 and 12 field plans shown in tables 1 (g) and (i) while all algorithms showed improved accuracy $(1 \%$ dev.) in the 9 fields plan as shown in table 1 (h). Table 2 shows results of the absorbed dose measured for different field plans with the bone inhomogeneity along with the percentage deviation from the reference dose $(1.00 \mathrm{~Gy})$ and the standard deviation for the 6 measurements taken for the 6 $\mathrm{MeV}$ photon beam. In tables 2 (a), (c) and (f), results of HB and FSS algorithms showed better accuracy (1 and $0 \%$ dev.) compared to others while convolution showed the least accuracy (4\% dev.). S, FSS and HB showed better accuracy $(1 \% \mathrm{dev}$.) in tables $2(\mathrm{e})$, however the accuracy of convolution improved in table 2 (d) for the 2 opposed field plans. HB was the only algorithm that showed improved accuracy $(2 \% \mathrm{dev}$.) in the wedged field as shown in table 2 (b) while S along with HB had the most improved accuracy ( 0 and $1 \%$ dev.) in the 6 and 12 field plans as shown in table $2(\mathrm{~g})$ and (i). All algorithms showed improved accuracy (1\% dev.) in the 9 fields plan as it was in the solid water measurements as shown in table $2(\mathrm{~h})$. There is a good standard deviation between the measurements for all plans. Table 3 shows the result of the absorbed dose measured with the lung inhomogeneity along with the percentage deviation from the reference dose $(1.00 \mathrm{~Gy})$ and the standard deviation between the 6 measurements taken for the $6 \mathrm{MeV}$ photon beam. FSS and HB showed better accuracy ( $1 \%$ dev.) in tables 3 (a), (b), (c) compared to C and S. Only the HB algorithm had a good accuracy $(-1 \%$ dev.) in the opposed field plan as observed in table 3 (d) while all algorithms had good accuracy ( 1 and $2 \%$ dev.) in the 3 and 4 fields plan as shown in tables 3 (e) and (f). S and HB had a better accuracy (0 and $1 \%$ dev.) in the 6 , 9 and 12 fields plan as shown in table $3(\mathrm{~g})$, (h) and (i) respectively.

Table 5 shows the result of the absorbed dose measured in solid water along with the percentage deviation from the reference dose $(1.00 \mathrm{~Gy})$ and the standard deviation between the 6 measurements taken for the $18 \mathrm{MeV}$ photon beam. FSS and HB showed better accuracy ( 1 and $2 \%$ dev.) in tables 5 (b) and (d) for the wedge and parallel opposed fields. The C, S and HB algorithms were better for the 12 field plan as shown in table 5 (i) while $\mathrm{C}$ and FSS showed improved accuracy (1\% dev.) for the 4 field and oblique field plans in table 5 (c) and (f). The $\mathrm{C}$ algorithm had better accuracy ( 0 and $1 \%$ dev.) in the 6 and 9 field plans as shown in table 5 $(\mathrm{g})$ and $(\mathrm{h})$ while FSS showed improved accuracy $(1 \% \mathrm{dev}$.) in the single and 3 field plan as shown in table 5 (a) and (e) respectively. Table 6 shows result of the absorbed dose measured for different field plans with the bone inhomogeneity in positions along with the percentage deviation from the reference dose (1.00 Gy) and the standard deviation for the 6 measurements taken for the $18 \mathrm{MeV}$ photon beam. In tables 6 (d) and (i), results of FSS, S and HB algorithmsshowed better accuracy ( 1 and $2 \%$ dev.) while convolution showed the least accuracy (3\% dev.). S algorithm showed better accuracy (1\% dev.) in tables 6 (a),(c) and (f) while FSS had the most improved accuracy $(2 \% \mathrm{dev}$.) for the wedge field in table 6 (b). S along with HB had the most improved accuracy (1\% dev.) in the 3 and 9 field plans as shown in table 6 (e) and (h). FSS and S showed improved accuracy $(1 \%$ dev.) in the 6 fields plan as shown in table $6(\mathrm{~g})$. There is a good standard deviation between the measurements for all plans. Table 7 shows the result of the absorbed dose measured with the lung inhomogeneity along with the percentage deviation from the reference dose (1.00 Gy) and the standard deviation between the 6 measurements taken for the $18 \mathrm{MeV}$ photon beam. FSS and S showed better accuracy ( $1 \%$ dev.) in tables 7 (h) for the 9 fields plan. Only the HB algorithm had a good accuracy ( 1 and $2 \%$ dev.) in the opposed and wedge field plans as observed in table 7 (b) and (d) while FSS had good accuracy ( $1 \%$ dev.) in the 3 and 4 fields plan as shown in table 7 (e) and f. S and HB had a better accuracy ( $2 \%$ dev.) in the 6 fields plan as shown in table 7 (g).S algorithm had the best accuracy $(1 \% \mathrm{dev}$.) for the single and oblique field plan as shown in table 7 (a) and (c), while FSS, S and HB where better (1\% dev.) for the 12 fields plan shown in table 7 (i). In general, convolution algorithm had a better accuracy in the solid water results where there is no inhomogeneity while the other algorithms had better accuracies for the bone and lung inhomogeneity results.

The results for all plans using the 4 algorithms in both beams were within established limits (Van Dyk et al., 1993,Ahnesjo andAspradakis, 1999, Fraass et al., 1998) and follow similar trend to those of Butts et al. (Butts et al., 2001) where anthropomorphic phantom was used.The method and results of the hybrid algorithm also follow similar pattern to those of Kelly (Kelly, 2011) where the LBTE was used to compute neutron transport equation. Larger deviations observed with the convolution algorithm at the bone inhomogeneity could be due to unaccounted scattered radiation contribution from the inhomogeneous material by the algorithm (Animesh, 2005. Muralidhar, 2009). However, convolution is good in tables 1 and 5 where there are no inhomogeneities. There is a general improvement across the tables for all algorithms in the larger field plans while poor deviation is noticeable for the wedged field plans across board. This may be due to the inability of the algorithms to model the fluence calculation for wedges (Van Dyk et al., 1993. Van Dyk et al., 1997). There 
is a similar trend in the results of the FSS and S, this may be due to the similarity in the methods (collapse cone) both algorithms used for calculation.Other sources of uncertainties such as set-up, phantom and the detector could have as well contributed to the deviation.

Calculation time in a single or fewer fields are longer with the hybrid algorithm than the convolution and superposition as shown in tables 4 and 8. Larger fields and higher energies take longer time to calculate, as do phantoms containing larger amount of bones as observed in table 8 for the $18 \mathrm{MeV}$ photon beam results. Most of the hybrid calculation time is in solving for the scattered photon and electron fluencies, which are performed only once for all beams in a plan. As a result, hybrid calculation time scale varies weakly with the number of fields. However, convolution and superposition calculation times scale increase linearly with the number of fields. As a result, the relative calculation speed of the hybrid increases with increasing number of fields in a plan. For cases with larger numbers of fields (i.e., 6, 9, and 12 field plans, IMRT, Rapid Arc), Hybrid becomes significantly faster than other algorithms. The hybrid algorithm showed general improvement across the board in all plans and since it can be used with the original data requirements of the Xio treatment planning, no extra data is therefore needed for its implementation.

The hybrid dose calculation algorithm was developed to address the accuracy and speed requirements for modern techniques in radiation therapy including IMRT and Rapid Arc. The hybrid algorithm provides comparable accuracy in treatment planning conditions to bench marked algorithms such as the convolution, superposition and fast superposition as shown in the results. Validation has been performed to assure dose calculation accuracy in typical inhomogeneous phantom. This algorithm can therefore be employed in the calculation of dose in advance techniques such as IMRT and Rapid Arc by radiotherapy centres with multiple algorithm system because it is easy to implement.

\section{References}

[1]. Van Dyk J, Barnett RB, Cygler JE, Shragge PC. “Commissioning and quality assurance of treatment planning computers.”Int. J. Radiat. Oncol.Biol. Phys. 1993;26:261-273.

[2]. Van Dyk J. "Quality Assurance." In Treatment Planning in Radiation Oncology.Khan FM, Potish RA (Eds.). (Baltimore, MD: Williams and Wilkins). 1997;123-146.

[3]. Lewis EE, Miller WF. 1984. "Computational methods of neutron transport", New York Wiley publication.

[4]. Wareing TA, McGhee JM, Morel JE, Pautz SD. 2001. Discontinuous Finite Element Sn Methods on Three-Dimensional Unstructured Grids.Nucl. Sci. Engr., 138:2.

[5]. Wareing TA, Morel JE, McGheeJM. 2000. Coupled Electron-Proton Transport Methods on 3-D Unstructured Grids, Trans Am. Nucl. Soc.83.

[6]. Siebers JV, Keall PJ, Nahum AE, Mohan R. 2000. Converting absorbed dose to medium to absorbed dose to water for Monte Carlo based photon beam dose calculations. Phys. Med. Biol. 45:983-995.

[7]. Vassiliev ON, Wareing TA, McGhee J, Failia G. 2010. Validation of a new grid-based Bolzmann equation solver for dose calculation in radiotherapy with photon beams. Phys. Med. Biol. 55:581-598.

[8]. Eleckta oncology system limited. 2000. Premium therapy operators manual. Stockholm: Eleckta precise treatment publication.

[9]. Muralidhar KR, Murthy NP, Raju AK, Sresty N. 2009. Comparative study of convolution, superposition, and fast superposition algorithms in conventional radiotherapy, three-dimensional conformal radiotherapy, and intensity modulated radiotherapy techniques for various sites, done on CMS XIO planning system. J Med Phys. 34:12-22.

[10]. IAEA Technical Report Series 398. 2000. Absorbed dose determination in external beam radiotherapy: an international code of practice for dosimetry based on standards of absorbed dose to water. Vienna: IAEA publication. 1011-4289.

[11]. Butts JR, Foster AE. 2001. Comparison of commercially available 3-dimensional treatment planning algorithms for monitor unit calculation in the presence of heterogeneities. J. App. Cli. Med. Phy. 2;1526-9914.

[12]. Kelly CT. 2011. The Linear Boltzmann Transport Equation. Raleigh: North Carolina state university publication.

[13]. 13Ahnesjo A andAspradakis MM. 1999."'Dose calculations for external photon beams in radiotherapy," Phys. Med. Biol. 44, R99R155.

[14]. 14Fraass B,Doppke K, Hunt M, Kutcher G,Starkschall G, Stern R and Van Dyk J. 1998.American Association of Physicists in Medicine, Radiation Therapy Task Group 53: "Quality assurance for clinical radiotherapy treatment planning," Med. Phys. 25, 1773-1829. 